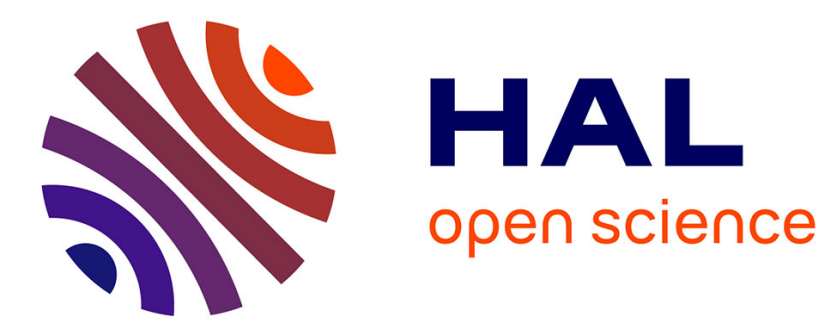

\title{
Experimental detection of upward-going cosmic particles and consequences for correction of density radiography of volcanoes
}

Kevin Jourde, Dominique Gibert, Jacques Marteau, Jean de Bremond d'Ars, Serge Gardien, Claude Girerd, Jean-Christophe Ianigro, D. Carbone

\section{To cite this version:}

Kevin Jourde, Dominique Gibert, Jacques Marteau, Jean de Bremond d'Ars, Serge Gardien, et al. Experimental detection of upward-going cosmic particles and consequences for correction of density radiography of volcanoes. Geophysical Research Letters, 2013, 40 (24), pp.6334-6339. 10.1002/2013GL058357 . insu-00944062

\section{HAL Id: insu-00944062 \\ https://hal-insu.archives-ouvertes.fr/insu-00944062}

Submitted on 10 Feb 2014

HAL is a multi-disciplinary open access archive for the deposit and dissemination of scientific research documents, whether they are published or not. The documents may come from teaching and research institutions in France or abroad, or from public or private research centers.
L'archive ouverte pluridisciplinaire HAL, est destinée au dépôt et à la diffusion de documents scientifiques de niveau recherche, publiés ou non, émanant des établissements d'enseignement et de recherche français ou étrangers, des laboratoires publics ou privés. 


\title{
Experimental detection of upward going cosmic particles and consequences for correction of density radiography of volcanoes
}

\author{
K. Jourde, ${ }^{1}$ D. Gibert, ${ }^{1,2}$ J. Marteau, ${ }^{3}$ J. de Bremond d'Ars, ${ }^{2}$ S. Gardien, ${ }^{3}$ C. Girerd, ${ }^{3}$ \\ J.-C. Ianigro, ${ }^{3}$ and D. Carbone ${ }^{4}$ \\ Received 16 October 2013; revised 3 December 2013; accepted 3 December 2013; published 19 December 2013.
}

[1] Muon tomography measures the flux of cosmic muons crossing geological bodies to determine their density. The telescopes used to perform measurements are exposed to noise fluxes with high intensities relative to the tiny flux of interest. We give experimental evidences of a so far never described source of noise caused by a flux of upward going particles. Data acquired on La Soufrière of Guadeloupe and Mount Etna reveal that upward going particles are detected only when the rear side of the telescope is exposed to a wide volume of atmosphere located below the altitude of the telescope and with a rock obstruction less than several tens of meters. Biases produced on density muon radiographies by upward going fluxes are quantified, and correction procedures are applied to radiographies of La Soufrière. Citation: Jourde, K., D. Gibert, J. Marteau, J. de Bremond d'Ars, S. Gardien, C. Girerd, J.-C. Ianigro, and D. Carbone (2013), Experimental detection of upward going cosmic particles and consequences for correction of density radiography of volcanoes, Geophys. Res. Lett., 40, 6334-6339, doi:10.1002/2013GL058357.

\section{Introduction}

[2] Density radiography with cosmic muons aims to determine the density of geological bodies by measuring the attenuation of the flux of cosmic muons caused by the screening effect of a known thickness of rock [Nagamine, 1995; Nagamine et al., 1995; Tanaka et al., 2001, 2005; Gibert et al., 2010; Lesparre et al., 2012c; Carloganu et al., 2013]. The most recent applications of muon density radiography of volcanoes concern monitoring of density variations [e.g., Tanaka et al., 2009; Shinohara and Tanaka, 2012], and high signal-to-noise ratio is required to measure tiny variations of the muon flux crossing the object of interest [Nagamine, 2003; Lesparre et al., 2010].

[3] Muon radiography uses telescopes counting and tracking particles coming from given directions [Lesparre et al.,

\footnotetext{
${ }^{1}$ Institut de Physique du Globe de Paris (CNRS UMR 7154), Université Sorbonne Paris Cité, Paris, France.

${ }^{2}$ Géosciences Rennes (CNRS UMR 6118), Université de Rennes 1, Rennes, France.

${ }^{3}$ Institut de Physique Nucléaire de Lyon, Université Claude Bernard, UMR 5822 CNRS, Lyon, France.

${ }^{4}$ Istituto Nazionale di Geofisica e Vulcanologia Sezione di Catania, Catania, Italy.

Corresponding author: D. Gibert, Institut de Physique du Globe de Paris (CNRS UMR 7154), Université Sorbonne Paris Cité, Paris, France. (gibert@univ-rennes1.fr)

(C)2013. American Geophysical Union. All Rights Reserved. 0094-8276/13/10.1002/2013GL058357
}

2010], and a main source of noise is due to fake tracks caused by independent particles that simultaneously hit the telescope detectors such that their impacts could falsely be interpreted as produced by a single particle crossing the detectors. In the present study, we give evidence of a new type of noise composed of upward going cosmic particles that may enter the back side of the telescope with trajectories identical to those of muons emerging from the volcano. Data acquired on Mount Etna and on La Soufrière of Guadeloupe [Lesparre et al., 2012c] reveal that upward going noise flux is present when the rear side of the telescopes is exposed to large and deep valleys. We discuss the impact of the measured upward noise on the quality of the density radiographies. Upward going particles are likely to be muons and high-energy electrons produced in the atmosphere volume located below the telescope level.

\section{The Telescopes}

[4] The telescopes are equipped with three detection matrices of $16 \times 16=256\left(5 \times 5 \mathrm{~cm}^{2}\right)$ pixels formed by intersecting scintillator strips [Lesparre et al., 2012a; Marteau et al., 2012]. The matrices are synchronized on the same master clock signal with a recently improved timing resolution of $1 \mathrm{~ns}$, and no shielding was used during these experiments.

[5] The orientation of the telescope is given by the azimuth, $\beta_{0}$, and the zenith angle, $\gamma_{0}$, of the telescope axis perpendicular to the matrices planes and oriented from rear to front. $\beta_{0}$ is measured from North and positive eastward, and $\gamma_{0}$ is positive and measured from the upward vertical axis to the telescope axis, i.e., $\gamma_{0}=90^{\circ}$ when the telescope axis is horizontal. We also use slopes $\alpha_{\mathrm{F}}$ in the forward direction and slopes $\alpha_{\mathrm{B}}$ in the backward direction, and measured positive upward so that negative slopes are assigned to telescope lines of sight looking below the horizontal plane.

[6] A trajectory is determined by the pixels crossed by the particle. Let $a_{i, j}$ be the front matrix pixel and $c_{k, l}$ be the rear matrix pixel where $i, k, j$, and $l$ take values between 1 and $N=16$. The combination of all possible pairs of pixels $\left(a_{i, j}, c_{k, l}\right)$ defines a set of $(2 N-1)^{2}=961$ discrete directions of sight $\mathbf{r}_{m, n}$ with $m=i-k$ and $n=j-l$ [Lesparre et al., 2012b; Marteau et al., 2012]. The angular range spanned by these directions is set by adjusting the distance $2 \times D$ between the front and rear matrices.

[7] One particle is considered detected when 1 pixel is fired on each matrix in a $20 \mathrm{~ns}$ time interval and their combination matches a linear trajectory. The raw data are corrected from the instrument acceptance and efficiency to obtain the absolute flux $\phi$ in $\mathrm{s}^{-1} \cdot \mathrm{sr}^{-1} \cdot \mathrm{cm}^{-2}$ [Lesparre et al., 2012b]. 


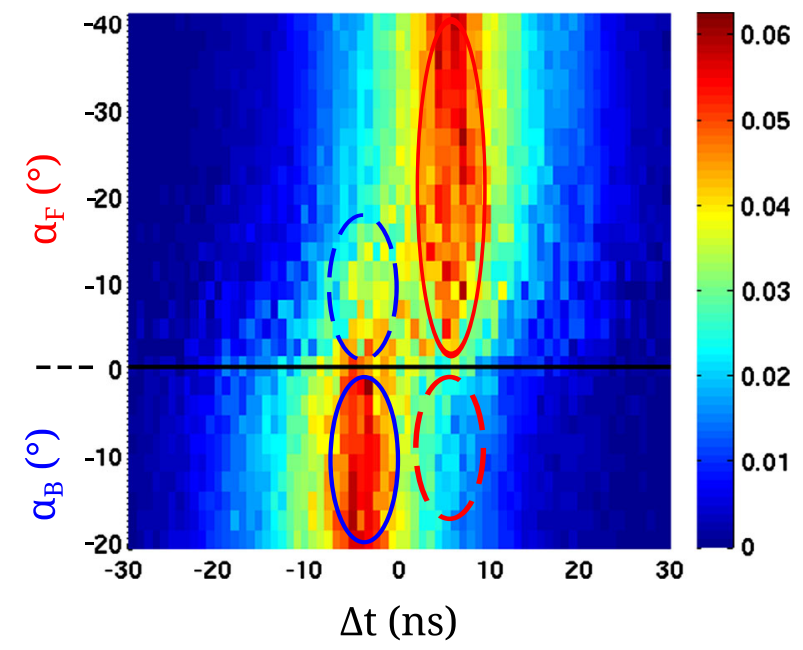

Figure 1. TOF distribution for the SMTOMO data set shown as normalized histograms as a function of zenith angle. The horizon is represented by the dashed line. The blue and red solid ellipses respectively show the backward $\left(\alpha_{B}<0\right.$ and $\Delta t<0)$ and forward $\left(\alpha_{F}<0\right.$ and $\left.\Delta t>0\right)$ events corresponding to the downward fluxes. The dashed ellipses show events corresponding to upward going events from forward (red ellipse, $\alpha_{B}<0$ and $\Delta t>0$ ) and backward (blue ellipse, $\alpha_{F}<0$ and $\left.\Delta t<0\right)$.

[8] Two experiments were performed on La Soufrière of Guadeloupe volcano [Lesparre et al., 2012a] and a third one on Mount Etna. Additional calibration data were acquired at Rennes 1 University (Britanny, France).

\section{Analysis Method}

[9] We distinguish the forward flux $\phi_{\mathrm{F}}$, whose particles enter in the telescope through the front matrix and escapes through the rear matrix, from the backward flux $\phi_{\mathrm{B}}$ whose particles enter through the rear matrix. These fluxes are separated into their upward going and downward going components respectively represented by $\phi_{\mathrm{F}, \mathrm{B}}^{u}$ and $\phi_{\mathrm{F}, \mathrm{B}}^{d}$ :

$$
\begin{array}{ll}
\phi_{\mathrm{F}}^{u}=\phi_{\mathrm{F}}\left(\alpha_{\mathrm{F}}>0\right) & \phi_{\mathrm{F}}^{d}=\phi_{\mathrm{F}}\left(\alpha_{\mathrm{F}}<0\right), \\
\phi_{\mathrm{B}}^{u}=\phi_{\mathrm{B}}\left(\alpha_{\mathrm{B}}>0\right) & \phi_{\mathrm{B}}^{d}=\phi_{\mathrm{B}}\left(\alpha_{\mathrm{B}}<0\right) .
\end{array}
$$

[10] A particle has a time-of-flight (TOF) $\Delta t=t_{\mathrm{C}}-t_{\mathrm{A}}$, where $t_{\mathrm{A}}$ and $t_{\mathrm{C}}$ are the detection dates in the front and rear matrices, respectively. Particles coming from forward have $\Delta t>0$, and backward particles have $\Delta t<0$ (experimental TOF can be seen in Figure 1. The theoretical unsigned TOF is given by

$$
\Delta t_{\text {theo }}(m, n)=c^{-1} \times \sqrt{4 \times D^{2}+\left(m^{2}+n^{2}\right) \times \delta^{2}},
$$

where $\delta=5 \mathrm{~cm}$ (pixel size) and $c$ is the light speed.

[11] The TOF probability density distribution, $\mathcal{P}(\Delta t)$, may be written as

$$
\mathcal{P}(\Delta t)=\mathcal{P}^{d}(\Delta t)+\mathcal{P}^{u}(\Delta t)
$$

where $\mathcal{P}^{d}$ and $\mathcal{P}^{u}$ respectively represent the distributions of the downward and upward TOFs. These distributions have the same normalized shape $\mathcal{F}$ (mean, std), as they share the same causes of uncertainties, but they have different amplitude and opposite mean. Consequently,

$$
\mathcal{P}(\Delta t)=r^{d} \mathcal{F}\left(\Delta t_{\text {theo }}, \sigma\right)+\left(1-r^{d}\right) \mathcal{F}\left(-\Delta t_{\text {theo }}, \sigma\right)
$$

where $0 \leq r^{d} \leq 1$ is the downward flux ratio equals to 0 for a pure upward going flux and to 1 for a pure downward going flux. The average of the measured TOFs reads as follows:

$$
\langle\Delta t\rangle=\Delta t_{\text {theo }} \times\left(r^{d}-\left(1-r^{d}\right)\right),
$$

and

$$
r^{d}=\frac{\langle\Delta t\rangle+\Delta t_{\text {theo }}}{2 \Delta t_{\text {theo }}} .
$$

[12] In the remaining we shall distinguish between the forward ratio, $r_{\mathrm{F}}^{d}=\phi_{\mathrm{F}}^{d} /\left(\phi_{\mathrm{F}}^{d}+\phi_{\mathrm{B}}^{u}\right)$ and the backward ratio, $r_{\mathrm{B}}^{d}=\phi_{\mathrm{B}}^{d} /\left(\phi_{\mathrm{B}}^{d}+\phi_{\mathrm{F}}^{u}\right)$.

[13] The processing sequence to obtain the upward flux is as follows:

[14] 1. For each direction $\mathbf{r}_{m, n}$, the measured TOFs $\Delta t$ are bootstrapped to get $\langle\Delta t\rangle$ (equation (5)) and its uncertainty;

[15] 2. The theoretical TOF is computed with equation (2);

[16] 3. The downward flux ratio $r^{d}$ is derived with equation (6) and, to further reduce the statistical uncertainty, $r^{d}$ is averaged over azimuths to be written as a function of zenith angle only. This simplification is an approximation when obstruction depends on the azimuth angle;

[17] 4. Finally, we recover

$$
\begin{aligned}
\phi^{d} & =r^{d} \times \phi, \\
\phi^{u} & =\left(1-r^{d}\right) \times \phi .
\end{aligned}
$$

[18] We emphasize that $\phi^{d}$ and $\phi^{u}$ cumulate uncertainties of both $r^{d}$ and $\phi$, while $r^{d}$ is a more accurate quantity to prove the existence of an upward going flux.

\section{Data Analysis}

\subsection{Field Measurement Sites}

[19] The Roche Fendue site is on the eastern side of La Soufrière lava dome at an altitude of $1268 \mathrm{~m}$ (Table 1, Figure 2a). The telescope was oriented horizontally to acquire the data set (RFCALIB) dedicated to upward going flux study. The front side of the telescope sees an open space free of rock obstruction down to $6^{\circ}$ below the horizon (Figure 2d). The backward landscape begins with a small horizontal plateau producing a rock obstruction of about $0.13 \mathrm{~km}$ that remains constant in $\left[0^{\circ} ; 6.8^{\circ}\right]$ and progressively decreases to zero at $5^{\circ}$ above the horizontal (Figure 2g).

[20] The Savane à Mulets site is located at the edge of a narrow plateau nearby the western side of La Soufrière lava dome at an altitude of $1189 \mathrm{~m}$ (Table 1, Figure 2b). The data set (SMTOMO), acquired during a high-resolution tomography experiment, merges three runs performed at the same location with different azimuth and zenith angles. The front side of the telescope sees a landscape occupied by the dome with an obstruction that varies from about $1 \mathrm{~km}$ at $0^{\circ}$ to more than $3 \mathrm{~km}$ at $4^{\circ}$ below the horizontal (Figure $2 \mathrm{e}$ ). The backward side of the telescope sees a wide open space 
Table 1. Site Characteristics and Main Acquisition Parameters ${ }^{\mathrm{a}}$

\begin{tabular}{lcccc}
\hline Data Set & $\begin{array}{c}\text { Rennes Lab } \\
\text { LBCALIB }\end{array}$ & $\begin{array}{c}\text { Roche Fendue } \\
\text { RFCALIB }\end{array}$ & $\begin{array}{c}\text { Savane à Mulets } \\
\text { SMTOMO }\end{array}$ & $\begin{array}{c}\text { Etna } \\
\text { ETOMO }\end{array}$ \\
\hline Altitude a.s.l. & $56 \mathrm{~m}$ & $1,268 \mathrm{~m}$ & $1,189 \mathrm{~m}$ & $3,095 \mathrm{~m}$ \\
$X_{\text {UTM }} / Y_{\text {UTM }}(\mathrm{m})$ WGS84 & $(30) 601,373 / 5,330,300$ & $(20) 643,347 / 1,774,036$ & $(20) 642,599 / 1,773,852$ & $(33) 499,007 / 4,178,852$ \\
Zenith angle $\gamma_{0}$ & $90^{\circ}$ & $90^{\circ}$ & $80^{\circ}-85^{\circ}-85^{\circ}$ & $85^{\circ}-80^{\circ}$ \\
Forward azimuth $\beta_{0}$ & $68.6^{\circ}$ & $215.5^{\circ}$ & $32^{\circ}-44^{\circ}-56^{\circ}$ & $110^{\circ}$ \\
Open space aperture & $0^{\circ}$ & $-7.4^{\circ}$ & $-7.4^{\circ}$ & $-8.0^{\circ} \dagger$ \\
$\quad$ below horizontal & & & & $-3.9^{\circ} \neq$ \\
Matrix distance $D$ & $85 \mathrm{~cm}$ & $85 \mathrm{~cm}$ & $60 \mathrm{~cm}$ & $85 \mathrm{~cm}$ \\
Axial acceptance $/$ Resolution & $5.8 \mathrm{~cm}^{2} \mathrm{sr} / 1.6^{\circ}$ & $5.8 \mathrm{~cm}^{2} \mathrm{sr} / 1.6^{\circ}$ & $11.5 \mathrm{~cm}^{2} \mathrm{sr} / 2.5^{\circ}$ & $5.8 \mathrm{~cm}^{2} \mathrm{sr} / 1.6^{\circ}$ \\
Acquisition time & $7 \mathrm{days}$ & $4 \mathrm{days}$ & $22-27-17$ days & $7-4 \mathrm{days}$ \\
\hline
\end{tabular}

${ }^{a}$ Note that for Etna site, apertures are for ranges of $15 \mathrm{~km}(\dagger)$ and $30 \mathrm{~km}(\ddagger)$. Also for the high definition data sets SMTOMO and ETOMO, we specify the characteristics of each subdata set when different.

with an obstruction varying from $0.1 \mathrm{~km}$ at $0^{\circ}$ to $0.3 \mathrm{~km}$ at $10^{\circ}$ (Figure 2h).

[21] The Etna site is located at an altitude of $3095 \mathrm{~m}$, about three times higher than La Soufrière sites (Table 1, Figure 2c). The back side of the telescope is directed toward a deep open space up to $8.0^{\circ}$ below the horizontal for distance up to $15 \mathrm{~km}$ (Figure 2i). For a range of $30 \mathrm{~km}$, the slope of the open space is reduced to $3.9^{\circ}$. The data set, called Етомо, merges the data for two acquisitions per- formed with a constant azimuth angle and slightly different zenith angles.

\subsection{Results for the Calibration Data Sets}

[22] The calibration data set LBCALIB acquired at the Rennes 1 University site is located on the third floor of building 15 of the Beaulieu campus, at an altitude of $56 \mathrm{~m}$ and $12 \mathrm{~m}$ above the ground. The topography is flat in a wide area of tens of kilometers around the telescope with
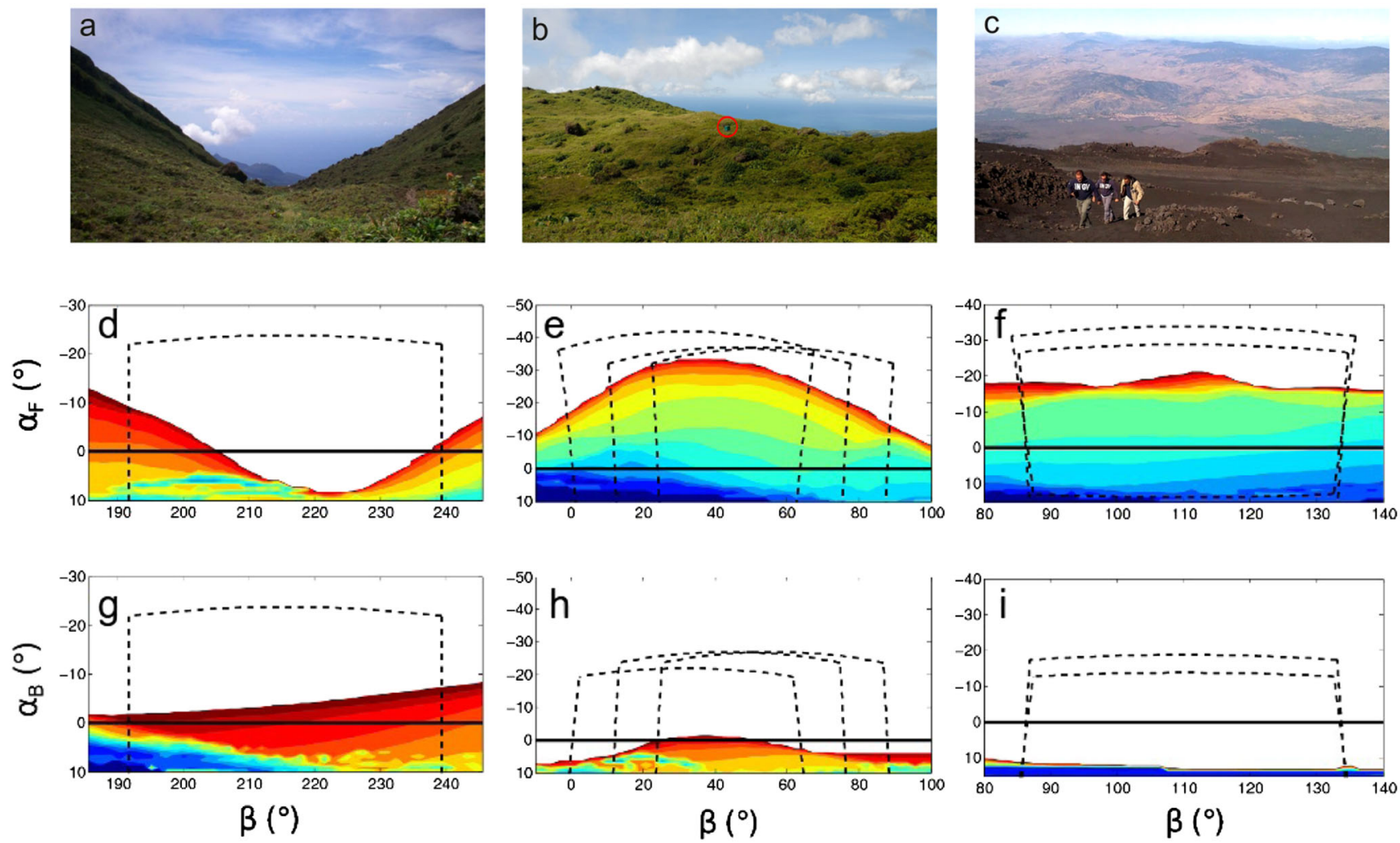

obstruction (m)

Figure 2. (top row) View of the unobstructed landscapes visible from the different measurement sites: (a) forward landscape at the Roche Fendue site; (b) backward landscape at the Savane à Mulets site (telescope circled in red); (c) backward landscape at the Etna North-East crater site. (middle and bottom rows) Rock obstruction (m) facing the telescope in the different acquisition configurations: (d,g) RFCALIB; (e,h) SMTOMO; (f,i) ETOMO. Rock obstruction is defined as the total rock thickness encountered along a given line of sight before it definitely enters into Earth. The telescope acquisition zone is delimited by a dotted black line. 
LBCALIB

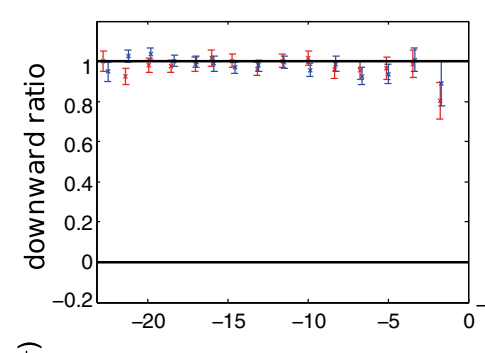

RFCALIB

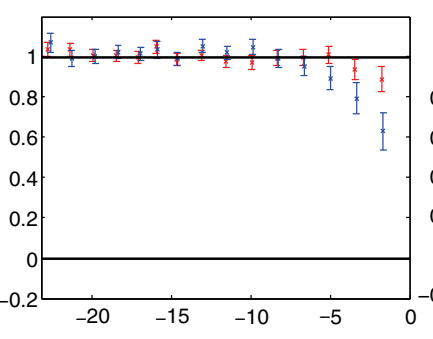

SMTOMO

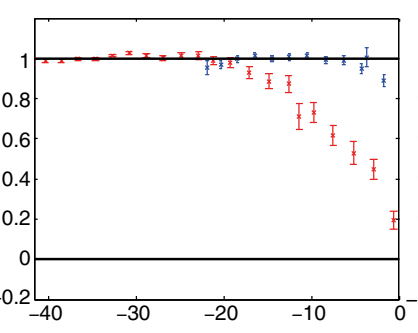

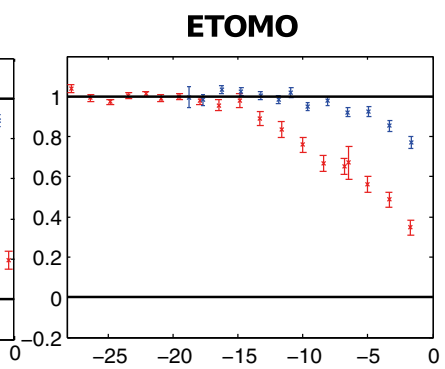
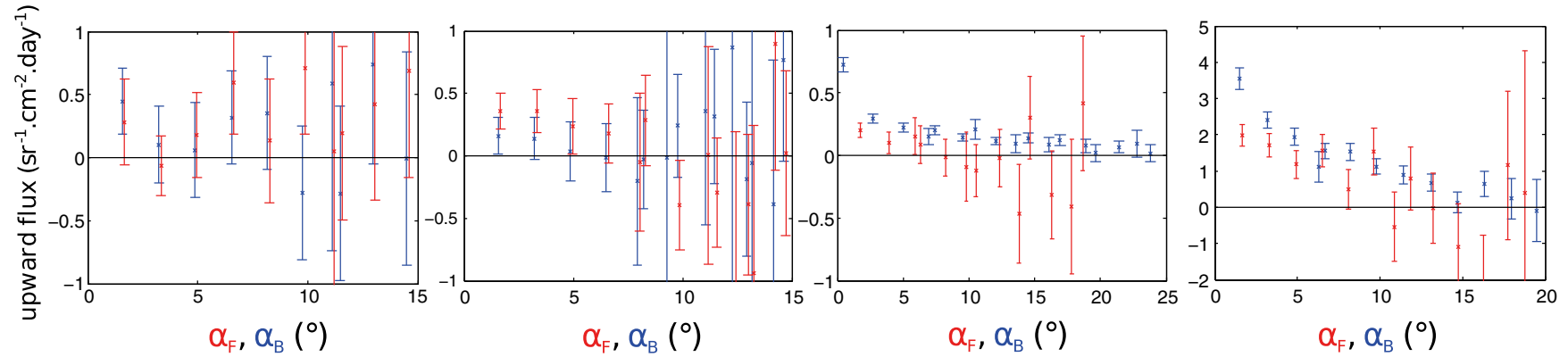

Figure 3. (top row, from left to right) Downward ratio $r_{\mathrm{F}}^{d}=\phi_{\mathrm{F}}^{d} /\left(\phi_{\mathrm{B}}^{u}+\phi_{\mathrm{F}}^{d}\right)$ (red) and $r_{\mathrm{B}}^{d}=\phi_{\mathrm{B}}^{d} /\left(\phi_{\mathrm{F}}^{u}+\phi_{\mathrm{B}}^{d}\right)$ (blue). (bottom row, from left to right) Upward fluxes $\phi_{\mathrm{F}}^{u}$ (red) and $\phi_{\mathrm{B}}^{u}$ (blue).

no open space aperture below the horizontal. The telescope was oriented horizontally to acquire the data set (labelled LBCALIB), and the horizontal lines of sight encompass a small solid angle symmetrically collecting all types of fluxes $\left(\phi_{\mathrm{F}}^{u}, \phi_{\mathrm{B}}^{u}, \phi_{\mathrm{F}}^{d}, \phi_{\mathrm{B}}^{d}\right)$, and making the distinction between upward and downward fluxes impossible (backward and forward fluxes remain distinguishable with the hightresolution clock).

[23] Both the downward ratio $r^{d}$ and the upward going flux $\phi^{u}$ derived from LBCALIB and RFCALIB are shown in Figure 3. For LBCALIB, $r^{d} \approx 1$ for all slopes except in a narrow range of $\alpha_{\mathrm{F}, \mathrm{B}}>-3^{\circ}$ above the horizon where $r^{d} \lesssim 1$ on both sides. This corresponds to a tiny upward flux of $0.3 \mathrm{sr}^{-1} \mathrm{~cm}^{-2} \mathrm{day}^{-1}$. The $r^{d}$ values obtained for RFCALIB are significantly less than 1 for $\alpha_{\mathrm{F}}>-5^{\circ}$ for the forward flux and for $\alpha_{\mathrm{B}}>-8^{\circ}$ for the backward flux. Backward $r_{B}^{d}$ is significantly lower than forward $r_{F}^{d}$ because the forward flux $\phi_{F}^{d}$ is stronger than the backward flux $\phi_{B}^{u}$. Forward $\phi_{F}^{u}$ takes values very close to LBCALIB on the first $5^{\circ}$ below the horizon. It is slowly decreasing down to $8^{\circ}$ below the horizon where important error bars, encompassing a null upward flux, forbid any reading (lack of statistics on $\Delta t$ ). Backward $\phi_{B}^{u}$ goes from $0.2 \mathrm{sr}^{-1} \mathrm{~cm}^{-2} \mathrm{day}^{-1}$ to 0 on the first $4^{\circ}$ below the horizon. The difference between forward and backward $\phi^{u}$ may be explained by the different obstruction patterns.

\subsection{Results for the Tomography Data Sets}

[24] For tomography measurements, the telescope has its main axis inclined toward the part of the volcano to be imaged with the largest acceptance, and the range of slopes spanned for the forward and the backward direction are different. The forward flux $\phi_{\mathrm{F}}^{d}$ is strongly attenuated by the volcano, leading to a lowering of the downward ratio. Tomography data sets have a good signal-to-noise ratio due to their long acquisition time. [Lesparre et al., 2010].

[25] Figure 1 presents the azimuthally averaged TOF $\Delta t$ of SMTOMO data subset (i.e., $\gamma_{0}=85^{\circ}$ and $\beta_{0}=44^{\circ}$ ). The resulting ratio is shown in Figure 3. The forward obstruction for this data subset goes from a few tens of meters at the upper edge of the volcano to $\approx 1 \mathrm{~km}$ for horizontal lines of sight (Figure 2e). Below the horizontal plane, obstruction quickly reaches $10 \mathrm{~km}$. The backward obstruction is null above the horizon and gradually increases up to a few hundred meters at $\alpha_{\mathrm{B}}=10^{\circ}$ (Figure 2h). The discrepancy between the forward and backward obstructions observed for the SMTOMO data is reflected in the corresponding $r^{d}$ curves (Figure 3, top row, third from left). The forward ratio $r_{\mathrm{F}}^{d}=\phi_{\mathrm{F}}^{d} /\left(\phi_{\mathrm{F}}^{d}+\phi_{\mathrm{B}}^{u}\right)$ (red curve) takes low values $0.2<r_{\mathrm{F}}^{d}<0.4$ for $0>\alpha_{\mathrm{F}}>-5^{\circ}$ and linearly grows to 0.9 at $\alpha_{\mathrm{F}} \approx-15^{\circ}$. The low $r_{\mathrm{F}}^{d}$ values indicate that the flux is mainly composed of upward going particles coming from backward directions. Conversely, the backward ratio $r_{\mathrm{B}}^{d}=\phi_{\mathrm{B}}^{d} /\left(\phi_{\mathrm{B}}^{d}+\phi_{\mathrm{F}}^{u}\right)$ (blue curve) does not significantly depart from 1 except at slopes just above the horizon where $r_{\mathrm{B}}^{d}=0.9$.

[26] The $r^{d}$ curves obtained for the Етомо data set (Figure 3, top row, rightmost) look very similar to the SMTOMO curves and reflect the obstruction asymmetry observed for this location (Figures $2 \mathrm{f}$ and $2 \mathrm{i}$ ). However, $r^{d}$ values significantly lower than 1 are obtained in the backward direction for $\alpha_{\mathrm{B}}>-5^{\circ}$, in agreement with the moderate forward obstruction for the corresponding range of slopes.

[27] The $r^{d}$ curves obtained from SMTOMO and ЕTOMO indicate the presence of an upward going flux $\phi^{u}$ in both the forward and the backward directions. However, this flux is always smaller in the forward direction because of the attenuation produced by the volcano obstruction. The backward upward flux $\phi_{B}^{u}$ strongly decreases in the first degrees (about $3^{\circ}$ for both SMTOMO and ETOMO) above the horizon and reaches zero at $20^{\circ}$ for SMTOMO and $15^{\circ}$ for ЕтOMO. Also, the flux (both upward and downward) on ETOMO is stronger than those on the other acquisition sites. The higher altitude of this site may be the reason for this difference, but detailed modelings of low-altitude atmospheric showers are necessary to document this issue. 


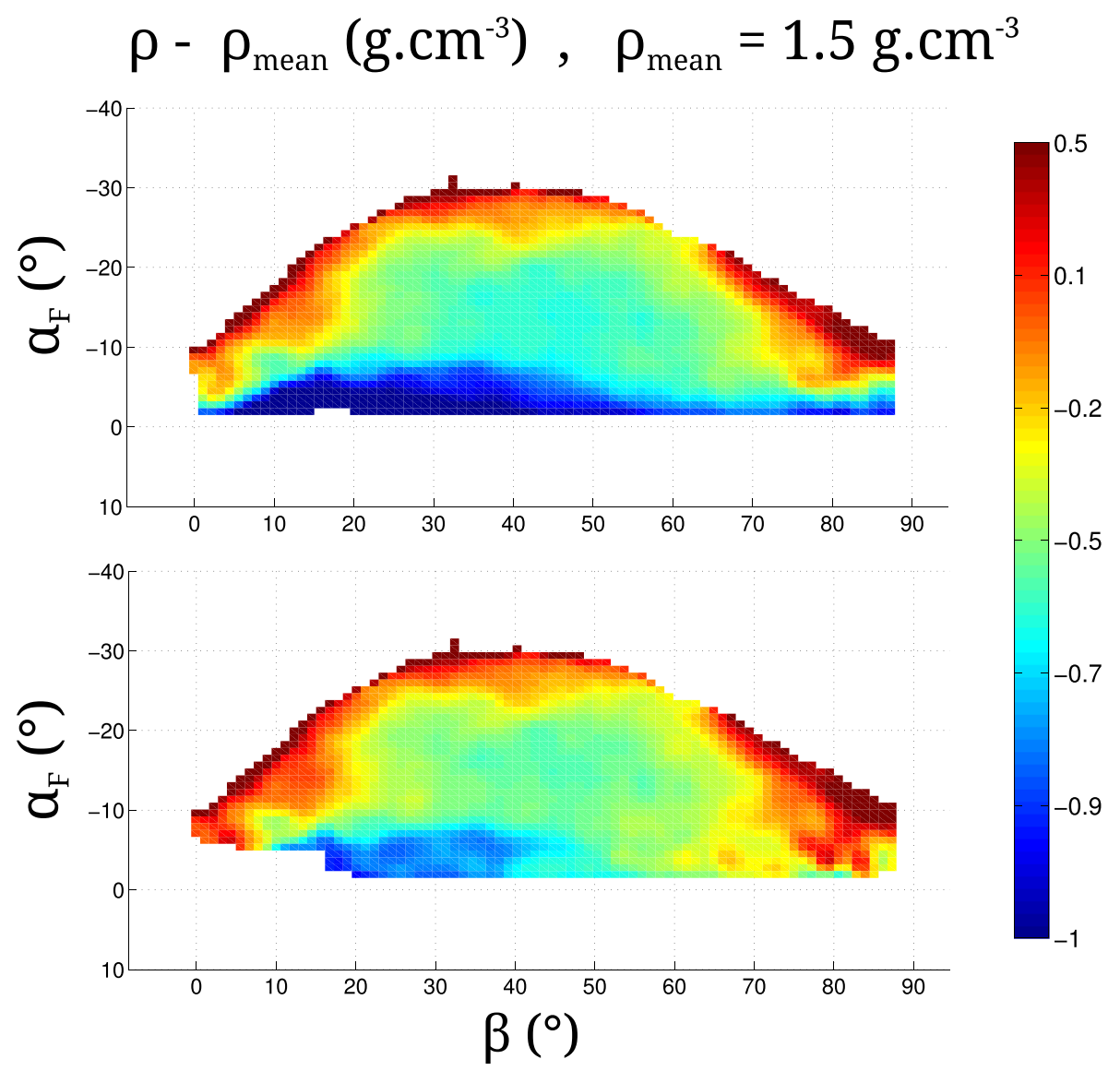

Figure 4. Tomography result of SMTOMO high-definition acquisition (top) without upward flux correction and (bottom) with upward flux correction. Rock density in $\mathrm{g} \mathrm{cm}^{-3}$.

\section{Influence of Upward Noise on Density Radiographies}

[28] The $r^{d}$ curves shown in Figure 3 clearly establish the existence of an upward going flux of particles whose relative intensity may be larger than the downward going flux of muons used to compute density radiographies. This produces a dramatic alteration of the density images for slopes just above the horizontal, and this is particularly regrettable since this concerns the deepest and generally most interesting parts of the studied volcano. A density radiography is obtained by computing the opacity, $\varrho_{m, n}\left[\mathrm{~g} \cdot \mathrm{cm}^{-2}\right]$, for each line of sight, $\mathbf{r}_{m, n}$, spanned by the telescope:

$$
\varrho_{m, n}=\int_{L_{m, n}} \rho(\xi) \mathrm{d} \xi=\bar{\rho} \times L_{m, n},
$$

where $\xi$ is the position along $\mathbf{r}_{m, n}$ of length $L_{m, n}$ across the volcano of density $\rho$. The opacity is converted into average density $\bar{\rho}_{m, n}=\varrho_{m, n} / L_{m, n}$ to construct the radiography image.

[29] The opacity in equation (8) is determined by searching the cutoff energy $E_{\min }(\varrho)$ that reproduces the measured flux of muons, $\phi_{m, n}$, by integrating the incident differential flux $\Phi_{0}\left[\mathrm{~cm}^{-2} \mathrm{sr}^{-1} \mathrm{~s}^{-1} \mathrm{GeV}^{-1}\right]$,

$$
\phi_{m, n}=\int_{E_{\min }\left(\varrho_{m, n}\right)}^{\infty} \Phi_{0}(E, \gamma) \mathrm{d} E\left[\mathrm{~cm}^{-2} \mathrm{sr}^{-1} \mathrm{~s}^{-1}\right] .
$$

Models for $\Phi_{0}$ are discussed by Lesparre et al. [2010] and, in the present study, we use the model given by Tang et al.
[2006]. Equation (9) shows that a positive bias in $\phi_{m, n}$ inducts an underestimate of $E_{\min }$, hence of opacity $\varrho_{m, n}$.

[30] To illustrate the importance of the bias produced by an upward going flux on reconstructed opacity images, we apply an upward flux correction to the SMTOMO data set. Computing $r^{d}$ for each $\mathbf{r}_{m, n}$, we use equation (7a) to obtain $\phi_{m, n}^{d}$ to be used in equation (9) instead of the total flux $\phi_{m, n}$.

[31] The upper part of Figure 4 shows the radiography obtained by using $\phi_{m, n}$ in equation (9), and the bottom part of the figure shows the corrected radiography obtained by using $\phi_{m, n}^{d}$. The low density region observed for $-10^{\circ}<\alpha_{F}<0^{\circ}$ in the biased radiography is strongly reduced both in intensity and extent in the corrected radiography. In particular, higher densities are obtained in the right-lower part (i.e., South region of the lava dome) of the corrected radiography in full agreement with other available data. The low density region that persists in the left-lower region of the corrected radiography agrees with electrical impedance tomography [Nicollin et al., 2006] that indicates the presence of cavities and hydrothermal reservoir in this part of the lava dome. The densities obtained after upward going flux correction remain low in absolute value, and this may indicate that further improvements of open sky flux models are necessary

\section{Conclusion}

[32] The data analyzed in the present study demonstrate the existence of a flux of upward going particles whose 
trajectories might be confounded with those of downward going muons crossing the volcano to radiography. The intensity of the upward going flux relative to the downward going flux may exceed $50 \%$ (Figure 3 ) and, if no distinction is made between these two types of fluxes, the resulting radiographies display underestimated densities in their bottom part for slopes $\alpha<15^{\circ}$ (Figure 4). High-resolution clocking systems are mandatory to acquire particle direction information allowing one to separate upward going and downward going fluxes in order to obtain reliable radiographies before performing a fine geophysical interpretation, at least in the first few degrees above the horizon. Upward going particles are likely to be created near the ground in the volume of atmosphere located below the altitude of the telescope. More dedicated field studies and atmospheric shower modelings are necessary to further document their origin.

[33] Acknowledgments. Guadeloupe experiments received the help from colleagues of the Volcano Observatory, the crews of the French Civil Security helicopter basis (http://www.helicodragon.com), and members of the National Natural Park of Guadeloupe (http://www.guadeloupeparcnational.fr). On-field maintenance of the telescope is ensured by Fabrice Dufour. Mount Etna experiments received the help of colleagues of the Volcano Observatory at Catania. We acknowledge the financial support from the UnivEarthS Labex program of Sorbonne Paris Cité (ANR-10LABX-0023 and ANR-11-IDEX-0005-02). This is IPGP contribution 3464. We are grateful to Tony Hurst and an anonymous reviewer who helped to improve the manuscript.

[34] The Editor thanks Tony Hurst and an anonymous reviewer for their assistance in evaluating this paper.

\section{References}

Carloganu, C., et al. (2013), Towards a muon radiography of the Puy de Dôme, Geosci. Instrum. Method. Data Syst., 2, 55-60.

Gibert, D., F. Beauducel, Y. Déclais, N. Lesparre, J. Marteau, F. Nicollin, and A. Tarantola (2010), Muon tomography: Plans for observations in the Lesser Antilles, Earth Planets Space, 62, 153-165.

Lesparre, N., D. Gibert, J. Marteau, Y. Déclais, D. Carbone, and E. Galichet (2010), Geophysical muon imaging: Feasibility and limits, Geophys. J. Int., 183, 1348-1361.
Lesparre, N., J. Marteau, Y. Déclais, D. Gibert, B. Carlus, F. Nicollin, and B. Kergosien (2012a), Design and operation of a field telescope for cosmic ray geophysical tomography, Geosci. Instrum. Method. Data Syst., 1, $33-42$.

Lesparre, N., D. Gibert, and J. Marteau (2012b), Bayesian dual inversion of experimental telescope acceptance and integrated flux for geophysical muon tomography, Geophys. J. Int., 188, 490-497.

Lesparre, N., D. Gibert, J. Marteau, J.-C. Komorowski, F. Nicollin, and O. Coutant (2012c), Density muon radiography of La Soufrière of Guadeloupe Volcano: Comparison with geological, electrical resistivity and gravity data, Geophys. J. Int., 190, 1008-1019.

Marteau, J., D. Gibert, N. Lesparre, F. Nicollin, P. Noli, and F. Giacoppo (2012), Muons tomography applied to geosciences and volcanology, Nucl. Instrum. Methods Phys. Res., Sect. A, 695, 23-28.

Nagamine, K. (1995), Geo-tomographic observation of inner-structure of volcano with cosmic-ray muons, J. Geogr., 104, 998-1007.

Nagamine, K. (2003), Introductory Muon Science, pp. 208, Cambridge Univ. Press, Cambridge, U. K

Nagamine, K., M. Iwasaki, K. Shimomura, and K. Ishida (1995), Method of probing inner-structure of geophysical substance with the horizontal cosmic-ray muons and possible application to volcanic eruption prediction, Nucl. Instrum. Methods Phys. Res., Sect. A, 356, 585-595.

Nicollin, F., D. Gibert, F. Beauducel, G. Boudon, and J.-C. Komorowski (2006), Electrical tomography of La Soufrière of Guadeloupe Volcano: Field experiments, 1D inversion and qualitative interpretation, Earth Planet. Sci. Lett., 244, 709-724.

Shinohara, H., and H. Tanaka (2012), Conduit magma convection of a rhyolitic magma: Constraints from cosmic-ray muon radiography of Iwodake, Satsuma-Iwojima volcano, Japan, Earth Planet. Sci. Lett., 349 , 87-97.

Tanaka, H., K. Nagamine, N. Kawamura, S. N. Nakamura, K. Ishida, and K. Shimomura (2001), Development of the cosmic-ray muon detection system for probing internal-structure of a volcano, Hyperfine Interact., $138,521-526$.

Tanaka, H., K. Nagamine, S. N. Nakamura, and K. Ishida (2005), Radiographic measurements of the internal structure of Mt. West Iwate with near horizontal cosmic ray muons and future developments, $\mathrm{Nucl}$. Instrum. Methods Phys. Res., Sect. A, 555, 164-172.

Tanaka, H., T. Uchida, M. Tanaka, H. Shinohara, and H. Taira (2009), Cosmic-ray muon imaging of magma in a conduit: Degassing process of Satsuma-Iwojima Volcano, Japan, Geophys. Res. Lett., 36, L01304, doi:10.1029/2008GL036451.

Tang, A., G. Horton-Smith, V. A. Kudryavtsev, and A. Tonazzo (2006), Muon simulations for Super-Kamiokande, KamLAND, and CHOOZ, Phys. Rev. D, 74, 053007 